\title{
Comparison of Several DPM Field Monitors for Use in Underground Mining Applications
}

\author{
Chelsea Barrett $^{1}$, Emily Sarver ${ }^{*}$, Emanuele Cauda ${ }^{2}$, James Noll ${ }^{2}$, Shawn Vanderslice ${ }^{2}$, \\ Jon Volkwein ${ }^{3}$ \\ ${ }^{1}$ Department of Mining and Minerals Engineering, Virginia Tech, Blacksburg, VA 24061, USA \\ ${ }^{2}$ CDC/NIOSH Pittsburgh Mining Research Division, Pittsburgh, PA 15236, USA \\ ${ }^{3}$ Science Consultant, Canonsburg, PA 15317, USA
}

\begin{abstract}
To improve worker health protection and support engineering applications in underground mines, such as ventilation-ondemand, capabilities are increasingly sought for continuous monitoring of diesel particulate matter (DPM). For near realtime monitoring over periods up to a full workshift, the FLIR Airtec handheld monitor was developed and calibrated to the NIOSH Standard Method 5040 measure of elemental carbon (EC), which is commonly used as an analytical surrogate for DPM. However, needs still exist for autonomous monitoring over longer periods (e.g., weeks to months). To meet those needs, two commercially available instruments are considered here, the Magee Scientific AE33 Aethalometer and the Sunset Laboratory Semi-continuous OC-EC Field Analyzer. Along with a prototyped monitor called the Airwatch, these were tested head-to-head against the Method 5040 EC and the Airtec in a controlled laboratory setting; and against one another in a field study at an underground mine. Key findings include: the OC-EC field analyzer performed well across a wide range of EC concentrations; the AE33 performed well at relatively low concentrations, but modifications or additional data corrections are likely needed at higher concentrations; and the Airwatch showed good potential, though significant improvements will be required if this instrument is to be further developed, including resolution of several mechanical issues and selection of an appropriate filter material and development of robust data corrections. Moreover, the relative advantages and disadvantages associated with each instrument (e.g., in terms of data quality, complexity and maintenance) must be considered in the context of the intended application and sampling environment.
\end{abstract}

Keywords: Diesel particulate matter; Occupational health; Continuous monitoring; Ultrafine aerosol; Underground mines.

\section{INTRODUCTION}

Diesel particulate matter (DPM) is the solid portion of diesel exhaust. These particulates occur primarily in the submicron range (Kittelson, 1998) and pose health hazards ranging from mild respiratory irritation to cancer risks (Ristovski et al., 2012). Underground miners generally experience the highest exposures of any occupational group due to the operation of heavy diesel equipment in confined spaces (MSHA, 2001). DPM is heterogeneous in nature and cannot be measured directly, but total (TC) or elemental carbon (EC) are often used as surrogate analytes because they represent primary DPM constituents. In metal/nonmetal mines in the United States, personal exposures to DPM are regulated

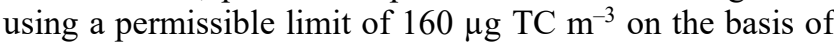
an 8-hour time-weighted average (TWA) (MSHA, 2006).

\footnotetext{
${ }^{*}$ Corresponding author.

E-mail address: esarver@vt.edu
}

For compliance purposes, TC is measured in filter samples using the NIOSH Standard Method 5040 (Birch, 2003). This is a thermal-optical method that results in quantitative measures of EC and organic carbon (OC), which can then be summed to determine TC. Samples are collected onto quartz fiber filters using a personal air pump, which is generally equipped with particle size selector(s) to limit collection of larger mineral dust particles. The sample analysis is then performed in a certified laboratory. This approach can also be used for non-compliance monitoring.

The significant lag time (i.e., on the order of weeks) between sample collection and Method 5040 results is problematic. Miners cannot take immediate action to limit their exposures, and it may be difficult to identify the circumstances surrounding overexposures retrospectively (Noll and Janisko, 2013). Engineering decisions related to DPM abatement are also delayed. Moreover, due to the nature of mining operations, DPM conditions may be very dynamic. This means that many data points, preferably on a higher-resolution time basis (e.g., one data point per minute or per hour as opposed to a point per day), are generally 
needed to correlate DPM trends with other conditions in the mine. Field monitoring capabilities that are (or near) continuous and real-time are needed to address these issues.

The Airtec handheld DPM monitor (FLIR, Nashua, NH) was specifically developed for tracking personal exposures in occupational environments, such as mines (Noll and Janisko, 2013). It uses a light extinction principle to determine EC as DPM accumulates on a filter. As the filter darkens during a given time increment, the decay in light transmittance is used to compute an absorbance value, which can then be correlated to the incremental increase in EC mass. The Airtec was specifically calibrated in the laboratory against Method 5040 samples in the wide concentration range around the permissible limit. The Airtec reports EC concentration $\left(\mu \mathrm{g} \mathrm{m}^{-3}\right)$ as a 5-minute rolling average, and it also reports TC concentration using an assumed ratio of TC $=1.3 \mathrm{EC}$ (Janisko and Noll, 2008). While it is a very useful tool for personal monitoring and periodic DPM surveying, the Airtec's design (i.e., with a filter that must be replaced regularly) is really not compatible with long-term continuous monitoring needs.

Long-term monitoring can provide insight into overall DPM behavior within an area or after a ventilation change. The first of three monitors which could be used for longterm area sampling that were investigated is the AE33 Aethalometer (Magee Scientific, Berkeley, CA). This is a commercially available instrument that was developed for continuous environmental monitoring applications, but it has been demonstrated in a limited number of field tests in underground mines (Barrett et al., 2017; Volkwein et al., $2017 \mathrm{a}, \mathrm{b})$. It also utilizes the light extinction principle and reports black carbon $\left(\mathrm{BC}, \mu \mathrm{g} \mathrm{m}^{-3}\right)$ on a 1-minute basis. Rather than using a single filter that must be replaced, the AE33 uses a self-advancing filter to allow autonomous operation over relatively long time periods (e.g., weeks to months). It has, heretofore, not been directly calibrated to Method 5040 EC in a laboratory setting. However, there have been a few field tests of this monitor in mines (Volkwein et al., 2017b) and an earlier model in other field settings (e.g., see Borak et al., 2003) that have shown different BC to EC ratios (i.e., between about 1.0 and 1.7). Thus, a direct calibration is important for interpreting AE33 field data against the standard EC measure.

The Airwatch was originally designed and prototyped as a field monitor intended specifically for high-DPM environments (Nomadics Inc., 2013). It was conceived as a continuous version of the Airtec handheld monitor and operates similarly to the AE33 with a self-advancing filter tape. The Airwatch outputs raw optical sensor voltage values, which can be translated into incremental or cumulative light absorbance values, and then used to calculate an EC concentration $\left(\mu \mathrm{g} \mathrm{m}^{-3}\right)$ using an absorbance-to-EC-mass calibration curve. Following brief testing of the original prototypes, several new and more rugged prototypes were built by Actinica (Boston, MA). One unit recently underwent preliminary calibration to Method 5040 EC mass and brief field-testing in an underground stone mine (Barrett et al., 2017). That testing indicated that the Airwatch could at times correlate well with EC mass concentration; however, its long-term performance has not been evaluated.
The Semi-Continuous OC-EC Field Analyzer (Sunset Laboratory Inc., Tigard, OR) is another potential option for DPM monitoring in mines, though it has never been tested in such a setting. This instrument, termed the "OC-EC field analyzer" from here on in this paper, is essentially a portable version of the Laboratory OC-EC Aerosol Analyzer used for the Standard Method 5040. The OC-EC field analyzer collects a sample on a filter that is housed within the analyzer furnace chamber, and then it runs the Method 5040 thermal-optical analysis to determine OC and EC mass on that filter, which can be converted to mass concentration values $\left(\mu \mathrm{g} \mathrm{m}^{-3}\right)$ using the sample collection time and flow rate (Sunset Laboratory Inc., 2014). The OC-EC field analyzer is considered semi-continuous as it produces one data point for every sample collection and analysis time increment. The sample collection time and flow rate are user defined, and the analysis time is about 20 minutes. As with the laboratory OC-EC analyzer, the OC-EC field analyzer requires high-purity gases for the thermal analysis and instrument calibration; in a field setting, these are supplied from cylinders. Since the same sample filter is used repetitively, build-up of refractory material can occur (e.g., due to the collection of mineral dust particles, which do not burn off like EC or OC), and thus the sample filter must be periodically replaced. Despite the more complex nature of the OC-EC field analyzer (versus the light-based monitors), its principle of measurement is effectively identical to that of the Standard Method 5040.

Notably, there are other instruments that have been used or suggested for occupational DPM monitoring, including light-scattering and condensation particle counters with capabilities in the submicron size range (e.g., Yu et al., 2015). Such instruments are not specific to DPM, however, and the propensity for relatively high dust concentrations in mines may limit their general applicability in these environments.

In order to evaluate and compare the available and indevelopment options for continuous DPM-specific monitoring, this study tested the AE33, Airwatch, and OC-EC field analyzer head-to-head in both the laboratory and the field. The Airtec monitor was also included in laboratory tests for comparison.

\section{EXPERIMENTAL DETAILS}

\section{Laboratory Testing}

A total of 19 laboratory tests were conducted in a Marple chamber at the National Institute for Occupational Safety and Health (NIOSH) Diesel Aerosol lab at the Bruceton Research Center in Pittsburgh, PA. The chamber is a large (approximately $1.8 \mathrm{~m}^{3}$ ) calm air chamber specially designed to limit spatial variability in aerosol distributions, such that side-by-side measurements can be conducted under carefully controlled and stable conditions (Marple and Rubow, 1983). This chamber has been used for a variety of experiments investigating dust and DPM and for calibration of monitoring equipment (Volkwein et al., 2006; Cauda et al., 2012; Noll et al., 2013). Particulates are generally fed to the chamber from the top, along with dilution/make-up air and exhausted 
from the bottom, with flow through the chamber being very slow. Due to its geometry, the chamber acts as a well-mixed reactor at mid-height where sampling occurs.

For this study, the DPM source was a Genset Tier-4 diesel engine (Kubota model D1703-M-BG-ET01) operated at either high (80\%) or low (20\%) load. Both types of conditions can be present in mine environments due to varying operating cycles for equipment (i.e., from idling to climbing steep grades with heavy material loads). A bleed-off of the engine exhaust was piped directly into the Marple chamber along with make-up air to achieve a relatively high $\left(\sim 600 \mu \mathrm{g} \mathrm{m}^{-3}\right)$, medium $\left(\sim 300 \mu \mathrm{g} \mathrm{m}^{-3}\right)$, or low $\left(\sim 100 \mu \mathrm{g} \mathrm{m}^{-3}\right)$ EC concentration. The EC concentration should be directly correlated to submicron DPM concentration here, and in most underground mine environments (Noll et al., 2007). In mines, high concentrations might represent peaks in an active section, and low concentrations might represent more typical background concentrations (e.g., see Noll et al., 2007; Gaillard et al., 2016).

Within each concentration range, tests were run for variable times to vary the amount of DPM mass sampled (Table 1). For Method 5040 analysis, the total sample mass is the important test design parameter and must be within the stated limits of quantification (Birch, 2003). The sample mass is used to determine a TWA EC mass concentration for the entire test period. Similarly, the semi-continuous OC-EC analyzer can be used to take one or a series samples during a given a test, which can be used to determine a TWA EC concentration over the respective sample collection time. For the continuous monitors using light extinction (i.e., AE33, Airwatch, and Airtec), the mass concentration inside the chamber is the important test design parameter. This parameter determines the incremental mass accumulation on the monitor filter, which is being measured at a particular frequency.

During each of the 19 tests, an AE33, Airwatch, OC-EC field analyzer, and five Airtec monitors were used to sample the DPM inside the chamber. All instruments were equipped with a size selector in order to limit collection of any oversized particles, which could significantly impact mass/light extinction measurements. Either a sharp-cut cyclone (per Cauda et al., 2014) or a combination of the Dorr-Oliver cyclone and diesel particulate matter impactor (SKC, Eighty Four, PA) (per MSHA, 2001) was used (see Table 2). Based on the instrument flow rates, the sharp-cut cyclone and DorrOliver cyclone/impactor combination should both produce a consistent cut size of around $0.8 \mu \mathrm{m}$. Standard filters or filter tape were used for the Airtecs (i.e., polytetrafluoroethylene, PTFE), AE33 (quartz fiber), and OC-EC field analyzer (quartz fiber). The Airwatch used a polyester/polyethylene tape, which was coated with a PTFE membrane $(0.3 \mu \mathrm{m}$ pore size).

The AE33 and Airwatch units tested here were the same units previously tested together in an underground stone mine (Barrett et al., 2017), and both were thoroughly cleaned prior to the experiments described here. The OC-EC field analyzer and all Airtecs were also cleaned prior to testing. Additionally, before and after each $~ 300$ total minutes of testing, the flow rate of each instrument was measured in order to allow for data adjustment where necessary (e.g., in case flow rate of any instrument drifted). For each test, the sample collection by each monitor was started/stopped simultaneously, and sample collection always began on a clean filter or filter spot. All sampling tube inlets were positioned at the same height in the chamber and in relatively close proximity to one another (i.e., no more than $7 \mathrm{~cm}$ apart).

For each test, a total of five Method 5040 samples were also collected-again, using sharp-cut cyclones and with

Table 1. Summary of laboratory test conditions.

\begin{tabular}{llllll}
\hline Test & $\begin{array}{l}\text { Target EC } \\
\text { Concentration } \\
\text { Range }\end{array}$ & $\begin{array}{l}\text { Engine } \\
\text { Condition } \\
(\text { load })\end{array}$ & $\begin{array}{l}\text { Target 5040 EC } \\
\text { Sample Mass } \\
(\mu \mathrm{g})\end{array}$ & $\begin{array}{l}\text { OC-EC Field Analyzer } \\
\text { Test Times } \\
(\mathrm{min})\end{array}$ & $\begin{array}{l}\text { Total Test } \\
\text { Time } \\
(\mathrm{min})\end{array}$ \\
\hline 1 & Low & High & 15 & 40 & 40 \\
2 & Low & Low & 170 & $50,50,50,53,52$ & 350 \\
3 & Low & High & 75 & $60,60,12$ & 170 \\
4 & Low & High & 125 & $60,60,60,48$ & 285 \\
5 & Low & Low & 50 & $50,50,12$ & 150 \\
6 & Low & Low & 10 & 30 & 30 \\
7 & Low & Low & 75 & $60,60,12$ & 170 \\
8 & Low & Low & 170 & $60,60,60,60,44$ & 360 \\
9 & Med & High & 10 & 10 & 10 \\
10 & Med & High & 50 & 4,19 & 41 \\
11 & Med & High & 125 & $22,14,8,22$ & 120 \\
12 & Med & High & 170 & $25,25,25,22$ & 154 \\
13 & Med & Low & 125 & $25,25,25$ & 113 \\
14 & Med & Low & 75 & 25,26 & 70 \\
15 & Med & Low & 50 & 30 & 45 \\
16 & Med & Low & 25 & 22 & 22 \\
17 & Med & High & 170 & $11,11,11$ & 92 \\
18 & High & High & 25 & 14 & 14 \\
19 & High & Low & 170 & $20,30,4$ & 90 \\
\hline
\end{tabular}


Table 2. Summary of equipment used in laboratory tests.

\begin{tabular}{lllll}
\hline Instrument & Units & $\begin{array}{l}\text { Flow Rate } \\
\left(\mathrm{L} \mathrm{min}^{-1}\right)\end{array}$ & $\begin{array}{l}\text { Cut Size } \\
(\mu \mathrm{m})\end{array}$ & Size selector \\
\hline Escort ELF pump (Method 5040 samples) & 5 & 2.2 & 0.8 & sharp-cut cyclone \\
Airtec & 5 & 1.7 & 0.8 & Dorr-Oliver cyclone/impactor \\
Airwatch & 1 & 2.2 & 0.8 & sharp-cut cyclone \\
AE33 & 1 & 2.0 & 0.89 & sharp-cut cyclone \\
OC-EC Field Analyzer & 1 & 2.2 & 0.8 & sharp-cut cyclone \\
\hline
\end{tabular}

tube inlets positioned near those of the monitoring instruments. The Method 5040 sample collection was done using a single vacuum pump with a five-way manifold to ensure precise sample collection times, which were started/stopped simultaneously with those of the monitoring instruments. Prior to sample collection, flow through each of the five critical orifice ports was measured to confirm they were all at the target flow rate $\left(2.2 \mathrm{~L} \mathrm{~min}^{-1}\right)$. The Method 5040 samples were collected onto pre-burned $37-\mathrm{mm}$ quartz fiber filters per the Birch (2003). The samples were later analyzed per the method using a Laboratory OC-EC Aerosol Analyzer (Sunset Laboratory Inc., Tigard, OR) in order to determine EC mass concentration $\left(\mu \mathrm{g} \mathrm{m}^{-3}\right)$ inside the chamber during each test.

To ensure that the EC concentration in the Marple chamber was near the target value and stable before each test, a tapered-element oscillating microbalance (TEOM) particulate monitor was used. It sampled from the same position and also used a sharp-cut cyclone to match the cut size (i.e., $0.8 \mu \mathrm{m}$ ) for other instruments. Since the only source of particulates for these tests was the engine exhaust, the TEOM measurements served as a direct proxy for mass concentration of DPM (i.e., which should be directly correlated to EC). Once the TEOM concentration stabilized near the desired concentration for a given test, no further adjustments were made in the exhaust to make-up air ratio for the entirety of that test period. TEOM data during the tests indicated that DPM concentrations were generally stable within a given test period (i.e., within $5 \%$ ), although some fluctuations were observed in particularly long tests, tests at low engine load, or those conducted at high concentrations.

\section{Field Testing}

While the laboratory tests were designed to evaluate the accuracy of each monitor relative to the Method 5040 EC under very controlled conditions, the objective of the field testing was to evaluate the overall performance of the monitors over relatively long periods of time. For this purpose, data were collected during five different weeks using the same AE33, Airwatch, and OC-EC field analyzer units tested in the laboratory, with the same sampling flow rates and cyclones (The short term measurement design of the Airtec precluded its use in field testing). On all occasions, the monitors were set up in the same stationary location (described below) such that their sampling inlets were side by side (i.e., no more than $10 \mathrm{~cm}$ apart) and oriented in the same direction. The OC-EC analyzer was programmed to collect 30-minute samples on the hour.

The field sampling was conducted in a large-opening underground stone mine near the main exhaust airway. The mine generally operates on a 5-day workweek (Monday through Friday), with a limited workforce on the weekends as needed. There are two eight-hour work shifts. Currently, maintenance and activities, such as roof scaling and blasthole drilling, are performed on the first shift, whereas most production activities occur on the second shift. Blasting is done following the second shift, allowing gases to clear overnight.

Due to the large openings in the mine, air velocities are generally low (less than $100 \mathrm{ft} \mathrm{min}^{-1}$ in most locations), and ventilation is provided by both natural and mechanical means. Natural ventilation forces fresh air into the mine primarily via the main ramp, and a blower fan also pushes fresh air into the mine through a dedicated airway. The main exhaust is through the belt slope, which extends from a midlevel of the mine just below the primary crusher to the surface. For effective ventilation, all air must travel through the main exhaust to exit the mine.

The primary airborne contaminant of concern in the mine is DPM (Gaillard et al., 2016). The mine's equipment fleet consists of several haul trucks, loaders, scalers, drills, and support equipment (such as light duty trucks). Even though DPM emissions generally only occur during the two daily work shifts, Monday through Friday, inactive workings in the mine can retain DPM in relatively stagnant air. These stagnant areas combined with the slow turnover rate of air, can allow significant background concentrations for hours or days after emissions stop. Previous monitoring studies near the main exhaust have shown a slow decline in EC over nights and weekends (Gaillard et al., 2016; Barrett et al., 2017; Volkwein et al., 2017a). Because EC near the main mine exhaust follows characteristic and expected patterns, all monitoring for the current study was performed in this location.

\section{Data Analysis}

As mentioned earlier, the instruments used in this study have different output variables based on their analytical techniques. Like the Method 5040, the OC-EC field analyzer reports a sample EC mass $(\mu \mathrm{g})$, which can be converted to a mass concentration $\left(\mu \mathrm{g} \mathrm{m}^{-3}\right)$ using the sample collection time and flow rate. The Airtec reports EC mass concentration $\left(\mu \mathrm{g} \mathrm{m}^{-3}\right)$ as a 5-minute rolling average. The AE33 reports BC concentration ( $\mathrm{ng} \mathrm{m}^{-3}$, converted to $\mu \mathrm{g} \mathrm{m}^{-3}$ here) as both a raw and corrected value, which is discussed further below. The Airwatch reports optical sensor voltage $(\mathrm{mV})$, which can be used to determine light extinction. (It is noted that light extinction on the Airwatch filter is primarily due to 
absorbance by the sample, rather than light scattering, and so the term absorbance is used from here forward.) Absorbance can be calculated using Eq. (1) for any given time increment, where $v_{f}$ is the final voltage and $v_{i}$ is the initial voltage. However, to translate absorbance to EC mass (or mass concentration in the sampling environment), a calibration curve must be used. In this study, the laboratory test results from the Airwatch were used to construct the calibration curve (i.e., using corresponding 5040-sample EC data), which was then applied to the field test results.

Absorbance $=-\log \left(\frac{v_{f}}{v_{t}}\right)$

In addition to having different output variables, the instruments used in this study collect data at different rates. Thus, some work was required to allow comparison of timeseries and TWA data. Fig. 1 shows example data from all instruments for two of the laboratory tests (i.e., one at low and one at medium concentration) to demonstrate several key points. First, the AE33, Airwatch, and Airtec data all appear as continuous (i.e., one data point per minute) over the test period shown. The AE33 and Airtec both record data on a 1-minute time basis; since multiple Airtecs were used for testing in this study, their data were averaged. The Airwatch records on a 5-second basis, however the data was condensed (by extraction) to a 1-minute basis to match the output from the AE33 and Airtec. In contrast, the OC-EC field analyzer data appear as semi-continuous. In tests where the instrument collected a series of samples (e.g., like tests shown in Fig. 1), this results in series of corresponding TWA
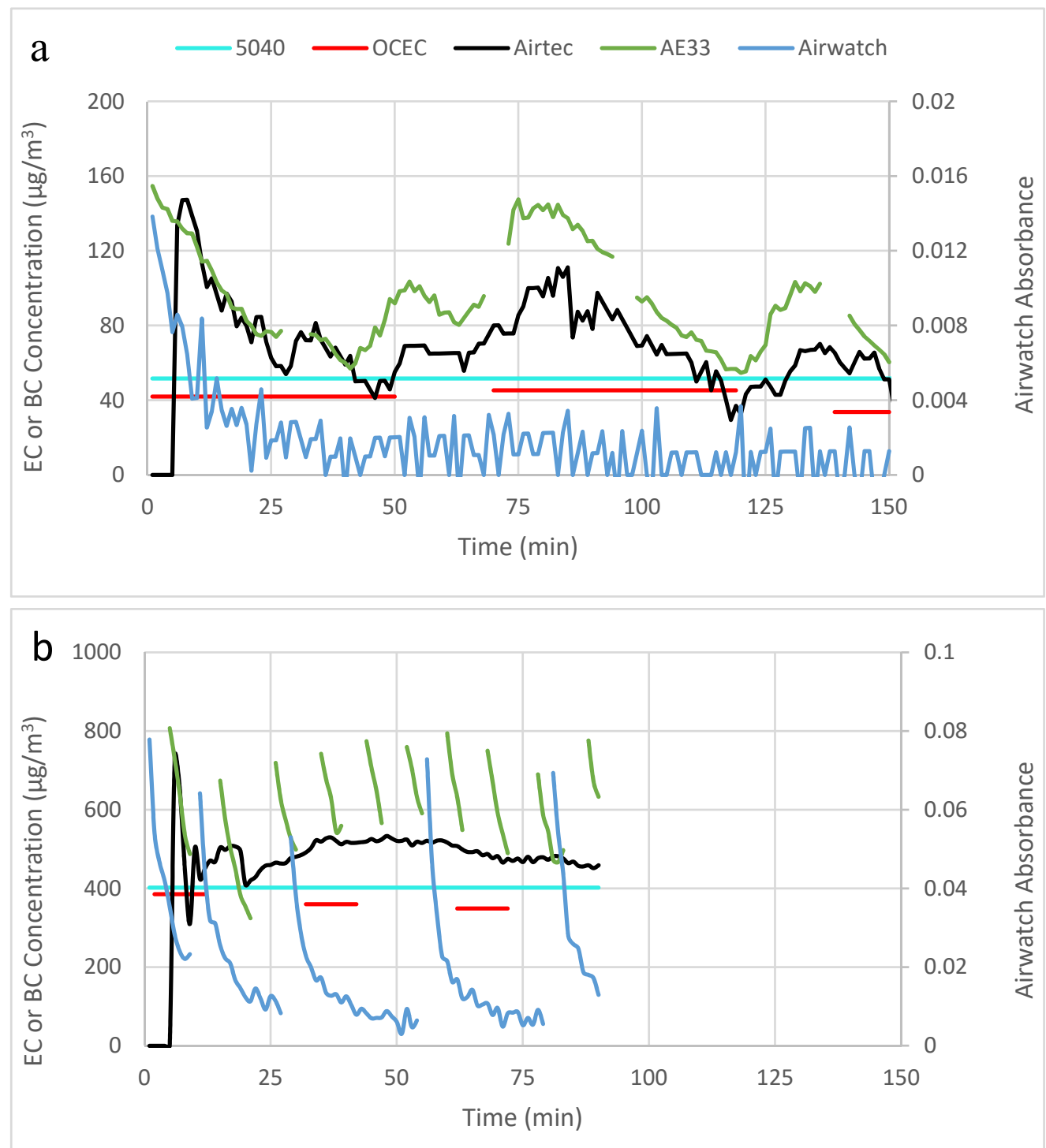

Fig. 1. Example data from each instrument collected in low (a) and medium (b) concentration laboratory tests. TWA EC concentrations are shown for Method 5040 samples and the OC-EC field analyzer; average EC concentration is shown for the Airtec; corrected (a) or raw (b) BC concentration is shown for the AE33; and calculated absorbance values are shown for the Airwatch. 
values. The Method 5040-sample value for each test appears as a single TWA of five replicate samples, which is applied over the entire test period.

Fig. 1 also illustrates breaks in data that correspond to filter tape advancement for the AE33 and Airwatch, or sample analysis by the OC-EC field analyzer (i.e., during which time sample collection is paused). The tape advance takes about 6 minutes for the AE33 and about 15 seconds for the Airwatch, and sample analysis takes about 20 minutes for the OC-EC field analyzer. To account for these breaks, data were interpolated between breaks (or extrapolated to the end of a test). Then, TWA values were computed for each filter spot (AE33 and Airwatch) or sample (OC-EC analyzer); and finally, an overall TWA for each test was computed using the filter spot or sample collection time as the weighting factor. For the Airtec, Fig. 1 shows that some time is required at the beginning of the test to reach a relatively stable value. This is partially because the instrument reports data on a 5-minute rolling average (i.e., only zero values are reported before minute 6). Moreover, the optical sensor in the monitor is also limited in detecting very small changes in light absorbance (i.e., incremental mass accumulation), which means that data stabilization can take longer in relatively low concentrations (Gaillard et al., 2016). Thus, TWA values for the Airtec were determined for the time period following data stabilization (i.e., after non-zero values were continually registered).

Another important observation from Fig. 1 is that the monitors which operate on a light extinction principle can exhibit filter loading effects - especially when sampling in high concentration environments. One type of effect is visible in the Airwatch absorbance data, which decline sharply at the beginning of each filter spot and then begin to stabilize. This sort of trend can be indicative of fibrous filter loading, which occurs in two distinct phases. First, sample mass is loaded in the spaces between fibers, and this produces a rapid decay in optical sensor voltage and thus light absorbance. Then, mass begins to load on top of the filter and produces a consistent unit of absorbance per unit of mass accumulated, which is the ideal behavior for a light extinction monitor in a constantconcentration sampling environment. A fibrous filter loading effect of this type was not necessarily anticipated for the Airwatch since the PTFE-coated filter tape used here was expected to load primarily on the surface. However, it is possible that the coating was degraded or that the pore size was significantly larger than the stated $0.3 \mu \mathrm{m}$ diameter, allowing penetration of the finest DPM particles. It is also possible that the Airwatch absorbance trend is somewhat influenced by light scattering as sample begins to load on the filter.

In order to process the Airwatch data from the laboratory tests and allow construction of the aforementioned calibration curve, absorbance values (i.e., calculated on a 1-minute basis per Eq. (1)) were only determined using data from "complete" filter spots (i.e., spots that were loaded to the point where the instrument self-advanced to a clean spot). This is because the relative influence of the initial drop in absorbance will be higher on spots that were only partially loaded; partially loaded filter spots were considered to be the last filter spot in tests with multiple spots or the only spot in tests with a single spot (i.e., the tape never advanced). Once the 1-minute absorbance values were determined for a given test, they were averaged to yield an overall 1-minute absorbance value for that test - and compared to the 1-minute EC mass value for that test, which was derived from the Method 5040 sample analysis (i.e., the total EC mass collected during the test divided by the total test time). This comparison for all tests was used to construct the Airwatch calibration curve.

The raw AE33 data shown in Fig. 1(b) (medium concentration test) exhibit another type of filter loading effect. The apparent decay in $\mathrm{BC}$ concentration, despite a relatively constant concentration in the test chamber, happens because particles may eventually start to load on top of one another (sometimes called "caking" or saturation), which reduces the unit light absorbance expected per unit of mass accumulation (Weingartner et al., 2003; Jimenez et al., 2007). Magee Scientific has sought to address this issue using a patented compensation method based on real-time tracking of the change in light absorbance on two filter spots collected simultaneously but at different flow rates (Drinovec et al., 2014). Using this "dual spot" method, the AE33 reports both a raw and corrected $\mathrm{BC}$ concentration value. While the method has been shown to yield accurate corrected data in relatively low concentration environments (e.g., urban air monitoring) (Drinovec et al., 2014), in the current study the AE33 did not consistently report corrected data in the medium and high concentration laboratory tests (tests 9-19 in Table 1). Thus, uncorrected (raw) data were analyzed for medium and high concentration tests, and corrected AE33 data were analyzed for the low concentration tests (e.g., like shown in Fig. 1(a)), and field testing.

It should also be noted that the saturation loading effect can potentially impact the Airtec and Airwatch too, if/when their filters begin to overload. It is difficult know whether any of the Airwatch behavior shown in Fig. 1 is due to this. However, the Airtec does not appear to be affected. Indeed, the Airtec was designed for continuous use over a full shift of personal monitoring in a mine, where a relatively wide range of EC masses (e.g., up to perhaps $250 \mu \mathrm{g}$ ) could be deposited on its larger 37-mm PTFE filter (Noll and Janisko, 2013). In Fig. 1(b), the total EC mass sampled was about 80 $\mu \mathrm{g}$.

\section{RESULTS AND DISCUSSION}

\section{Laboratory Testing}

Results of the Method 5040 analysis on all replicate samples from each test are reported in Table S1 (Supplemental Information). They confirmed that low, medium and high concentration tests had EC concentrations between about 55-165 $\mu \mathrm{g} \mathrm{m}^{-3}, 250-400 \mu \mathrm{g} \mathrm{m}^{-3}$, and around $600 \mu \mathrm{g} \mathrm{m}^{-3}$, respectively. No discernable difference was observed in the performance of any of the monitors based on the engine loading condition.

As mentioned earlier, the parameter of practical interest for the continuous monitors tested here is EC mass concentration, and so all results below are presented on this basis. However, some results are discussed in the context of 
mass since monitor performance issues (e.g., due to filter loading effects) may be fundamentally related to mass.

As expected, the OC-EC field analyzer results matched the standard Method 5040 results very well in the laboratory tests. Indeed, considering that the OC-EC field analyzer effectively uses the same thermal-optical method and is not subject to issues related to uniformity of particle deposition (i.e., since the entire filter area is analyzed), the monitor results in this study can generally be regarded as verification of the Method 5040 results. Fig. 2 compares the EC concentration results from the OC-EC field analyzer and the standard Method 5040 samples (all data tabulated in Table S2). Only one of 19 tests produced a data point that did not fit the trend of the other data. This statistical outlier (determined as a variance greater than three $\sigma$ away from the mean of the ratio between monitor and 5040 results) is shown as point A in Fig. 2. Inaccurate Method 5040 data are probably responsible for point $\mathrm{A}$, which was also found to be an outlier when comparing all other monitoring instruments to the Method 5040 results. Thus, the OC-EC field analyzer value was used for this data point instead of the Method 5040 value for evaluation of all other monitors.

The Airtec-derived TWA values also correlated well with the Method 5040 data (Fig. 3), which was expected since the Airtec is calibrated to the Method 5040 EC (Noll and Janisko, 2013; Janisko and Noll, 2008). A linear regression showed an $\mathrm{R}^{2}$ of 0.91 , a slope of 1.0 , and a y-intercept of $55 \mu \mathrm{g} \mathrm{m}^{-3}$. This fits reasonably well with field observations by Noll and Janisko (2013) in the same general EC ranges; overall, their data shows a slope of 0.93 and a y-intercept of $13 \mu \mathrm{g} \mathrm{m}^{-3}$. Thus, the Airtec had a slight positive bias, which was more pronounced in the lowest EC concentrations, but the bias diminished or even became slightly negative at higher EC concentrations - which is again consistent with the current study (Fig. 3). This change in the Airtec's performance across EC concentrations likely happens due to the aforementioned filter saturation effect, where particles eventually begin to load on top of one another. The effect is fundamentally an issue of mass accumulation rather than concentration in the sampling environment (i.e., it will happen once some total mass has been accumulated). For the tests conducted here, those with the highest sample masses (i.e., about $100 \mu \mathrm{g}$ EC or more) did in fact fall below the trend line for the rest of the data, actually producing more accurate (though less precise) Airtec results.

As mentioned earlier, the AE33 uses a dual-spot method to correct data for the filter loading effects. Here, the instrument only reported corrected $\mathrm{BC}$ concentrations consistently (i.e., for every filter spot within a test) for the low concentration tests (1-8). For these, the correlation between TWA BC concentration and Method $5040 \mathrm{EC}$ concentration values was good (i.e., $\mathrm{R}^{2}=0.81$, Fig. 4 ). The $\mathrm{BC}$ to $\mathrm{EC}$ ratio observed in these tests (i.e., 1.86, as indicated by the slope of the trend line in Fig. 4) is generally consistent with several anecdotal observations (1.5-2.0) in similar EC concentrations (Volkwein et al., 2017a, b), but it is much higher than the ratio (roughly 1.0) observed in another study where the EC concentrations were typically in the medium concentration range tested here (Barrett et al., 2017). In all of those studies, the AE33 was used to continuously monitor BC concentration in mine environments, which was periodically compared to Method 5040 EC concentration.

To examine the AE33 data across all 19 laboratory tests, the uncorrected $\mathrm{BC}$ concentration data were first used (Fig. S1). While the correlation to Method 5040 data over the entire EC concentration range was still fairly good $\left(\mathrm{R}^{2}\right.$ of 0.80 ), the apparent $\mathrm{BC}$ to $\mathrm{EC}$ ratio was quite low (slope of 0.88 ) - closer to the prior field study mentioned above in

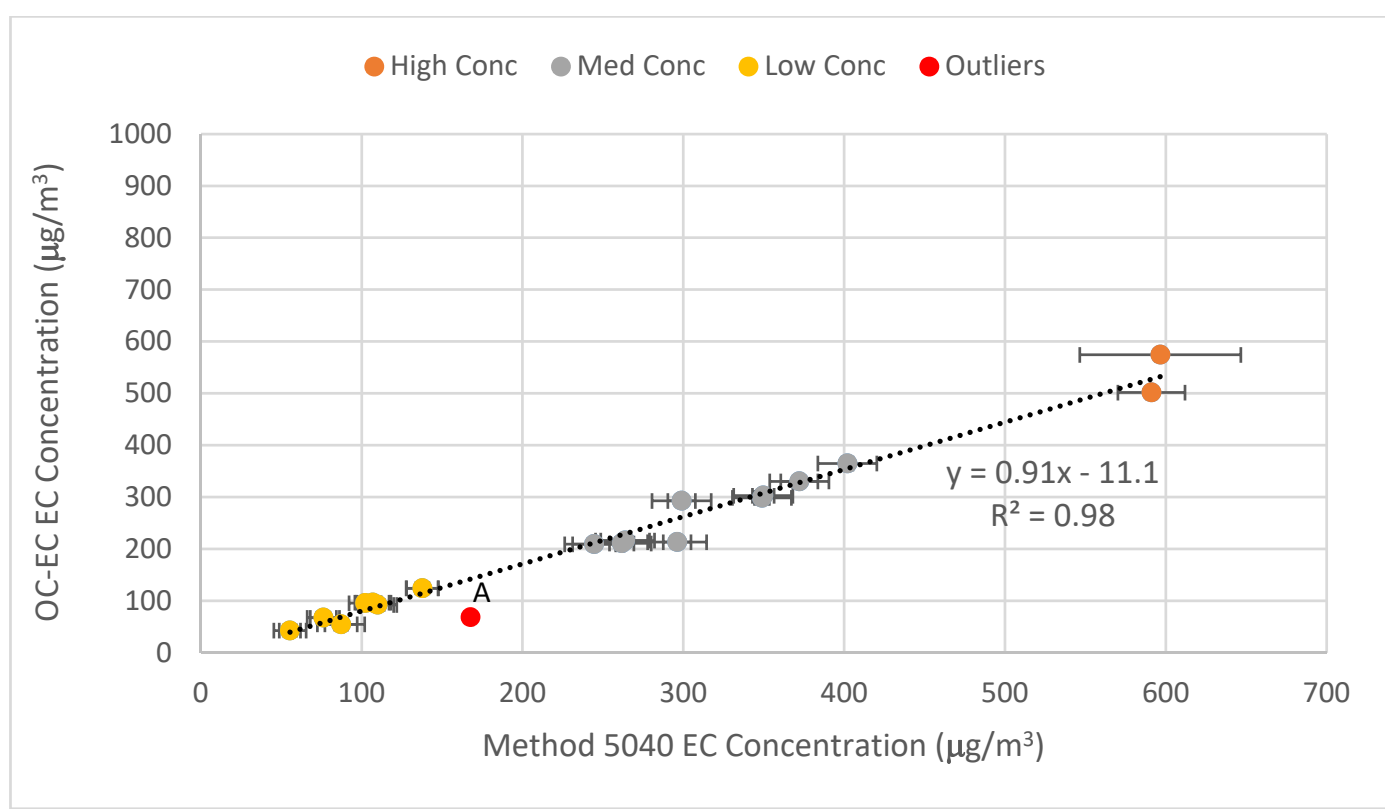

Fig. 2. OC-EC field analyzer EC concentration versus Method 5040 EC concentration for laboratory tests. Error bars represent $95 \%$ confidence intervals for replicate 5040 samples results; confidence intervals were not computed for OC-EC field analyzer data since individual samples collected within each test had variable collection times. 


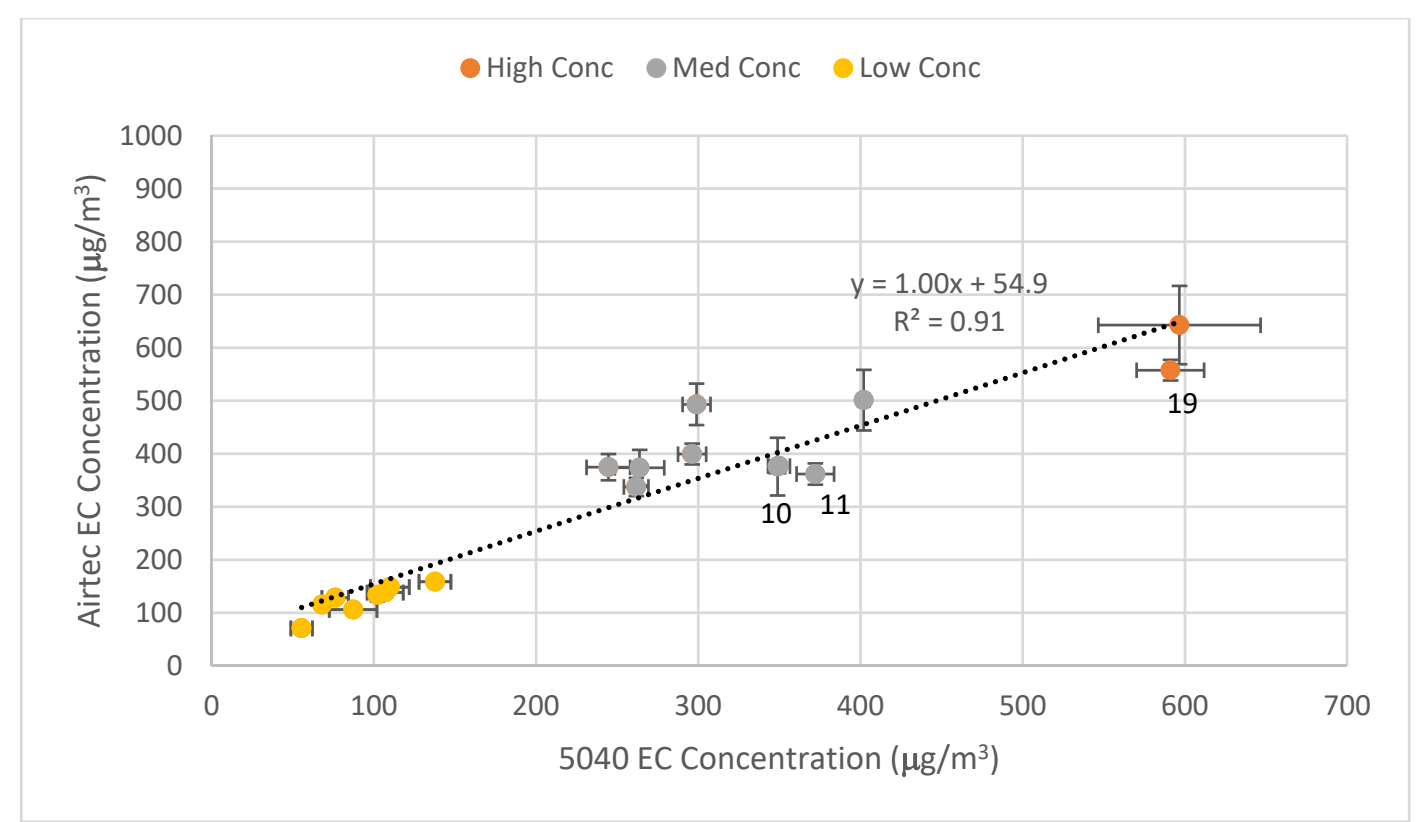

Fig. 3. Airtec EC concentration versus the Method 5040 EC concentrationfor laboratory tests. Error bars represent $95 \%$ confidence intervals for replicate 5040 samples and Airtec (5 monitors) results. Data points for tests 10, 11 , and 19 are labeled, and these tests had relatively high sample masses of 99,112 , and $123 \mu \mathrm{g}$, respectively.

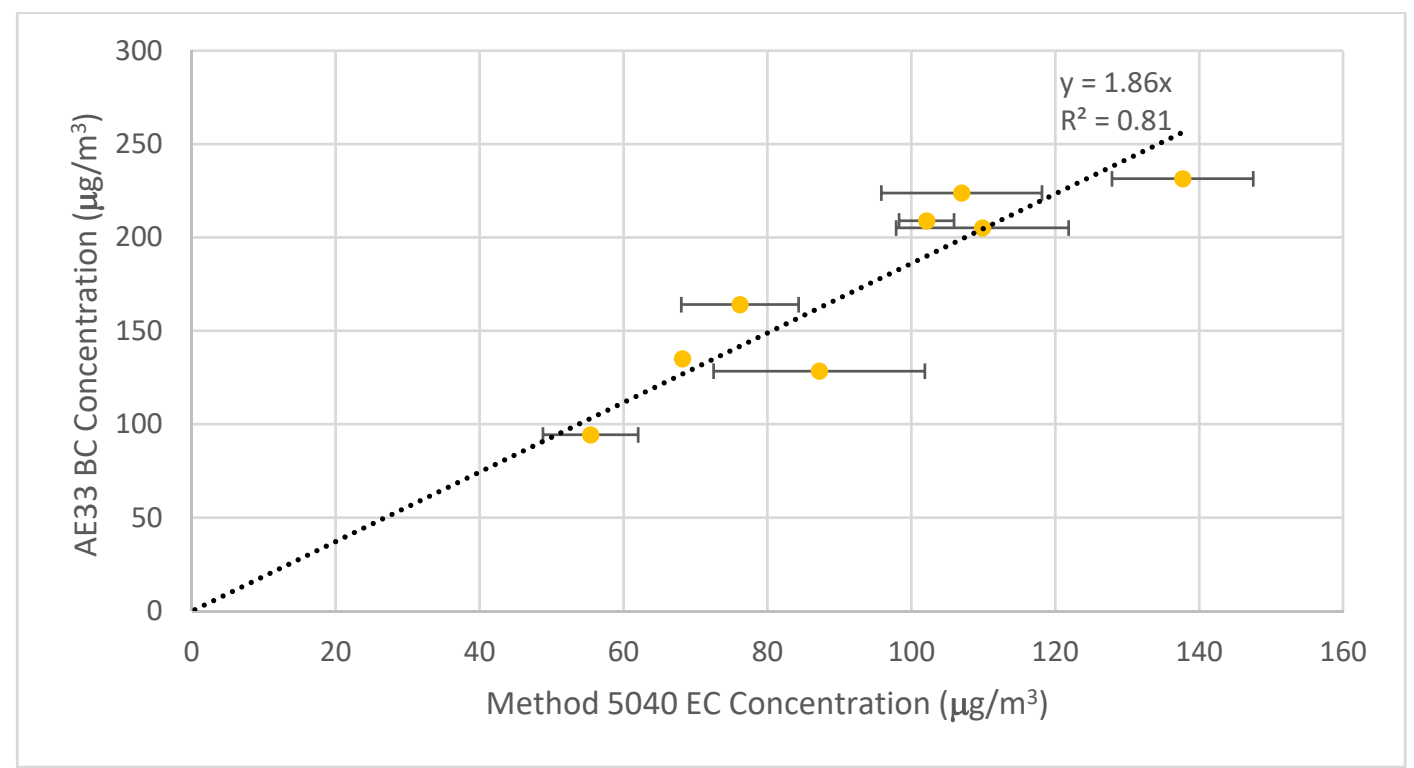

Fig. 4. Corrected AE33 BC concentration versus Method 5040 EC concentration for low concentration laboratory tests. Error bars represent $95 \%$ confidence intervals for replicate 5040 samples; confidence intervals were not computed for AE33 data since individual filter spots within each test had variable collection times.

medium EC concentrations (Barrett et al., 2017). This is consistent with expectations for the uncorrected data, since the saturation filter loading effect most severely impacts measurements in higher DPM concentrations (i.e., where the rate of mass accumulation on the filter is higher). This effect manifests as an underestimation of BC concentration (Drinovec et al., 2014)

To further explore this finding, the uncorrected AE33 data were also reduced to "first-minute" data, meaning that only the $\mathrm{BC}$ concentration measured during the first minute on each filter spot during a test was used to compute a TWA concentration for the test. This effectively focuses analysis on what should be the best data (i.e., the data least impacted by the saturation effect) under the test conditions here, but it also makes the AE33 more of a semi-continuous monitor. Fig. 5 shows the first-minute $\mathrm{BC}$ concentration. There is a clear change in the relationship with Method 5040 EC as concentration increases, producing a $\mathrm{BC}$ to $\mathrm{EC}$ ratio near 2.0 at low concentrations and a ratio near 1.5 at higher concentrations. These results more fully explain the 
previous observations of apparent variability in $\mathrm{BC}$ to $\mathrm{EC}$ ratio in different EC concentrations. Moreover, they suggest that some effect of filter loading is present even within the first minute of sample mass accumulation. Nevertheless, the first-minute data demonstrate the potential of the AE33 to predict $\mathrm{EC}$ from $\mathrm{BC}$ across a wide range of concentrations. To resolve the issue of filter saturation in higher concentration environments, simple modifications to the sampling train might be considered (e.g., to dilute the sample flow or reduce the the flow rate).

Regarding the Airwatch, as described earlier, a 1-minute time basis was used to analyze the data. Fig. 6 shows the resulting calibration curve, which plots the average 1-minute Airwatch light absorbance value against the corresponding 1-minute EC mass accumulation value in each test (i.e., derived from the 5040 sample results). There is a good correlation $\left(\mathrm{R}^{2}=0.73\right)$, even despite the non-ideal behavior in absorbance as a sample loads onto the Airwatch filter (e.g., as shown in Fig 1). Notably, the slope of the trendline in Fig. 6 is significantly greater than that yielded by data from a previous calibration of the same Airwatch unit (see Fig. S2, generated using raw data from the Barrett et al., 2017 study). In the previous work, the Airwatch was also tested in the Marple chamber with constant, albeit only low,

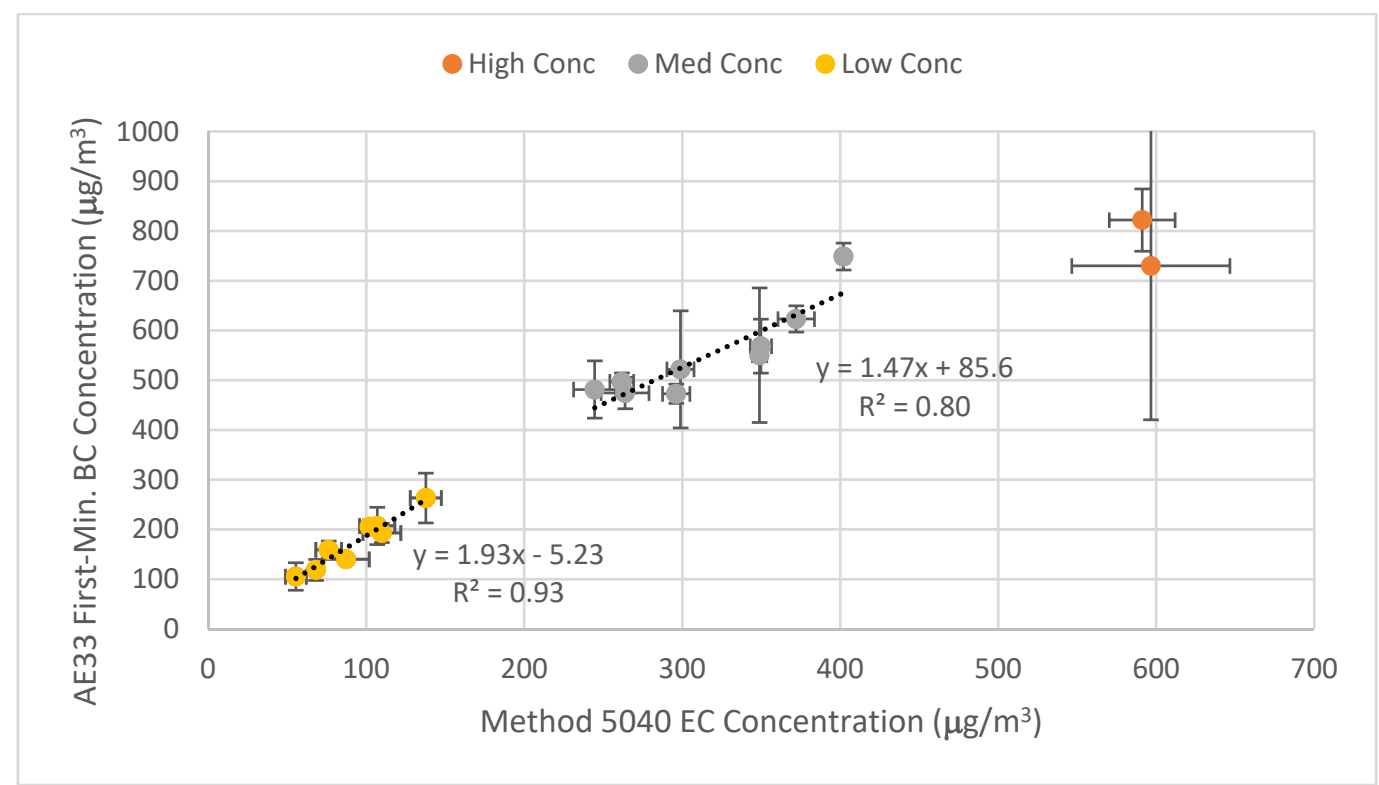

Fig. 5. First-minute AE33 BC concentration versus Method 5040 EC concentration for laboratory tests. Error bars represent $95 \%$ confidence intervals for replicate 5040 samples and first-minute data from all AE33 filter spots in each test.

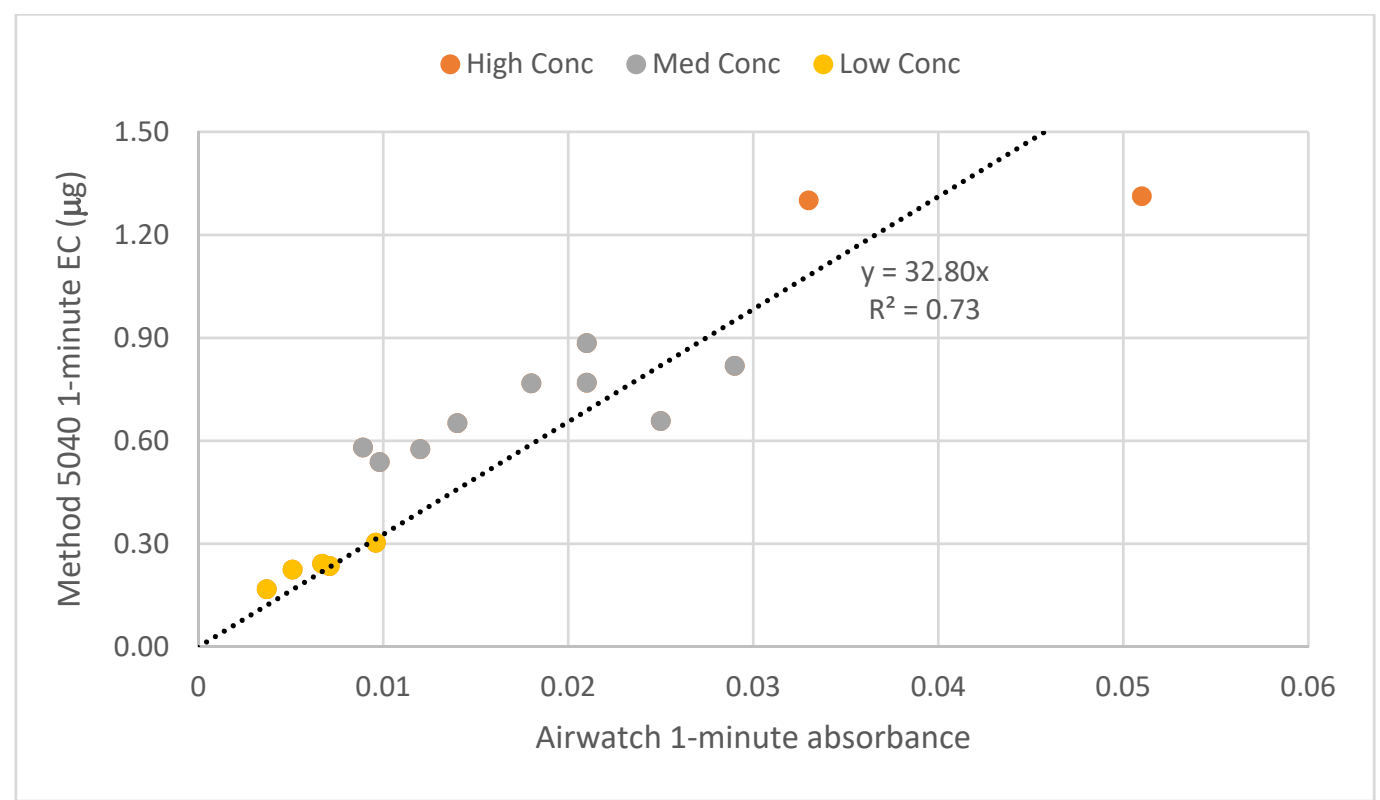

Fig. 6. Airwatch calibration curve showing average EC mass accumulated on filter per minute versus average light absorbance per minute. 
EC concentrations; and the general absorbance trend seen in Fig. 1(a) was also observed. However, the test durations were relatively short such that the Airwatch filter tape was not allowed to advance, and so the Barrett et al. (2017) data represents only partial filter spots. As explained above, partial filter spots are more affected by the characteristically high absorbance values observed during early sample collection on each spot.

It is also worth noting that the starting optical sensor voltage of the Airwatch varied between the Barrett et al. (2017) study and the work reported here. In the earlier work, the starting voltage on a clean filter spot was consistently between about 3.2-3.8 mV, whereas in the current laboratory and field tests it was between about $2.0-2.5 \mathrm{mV}$. While the starting voltage should theoretically not affect the voltage decay trend (and thus computed absorbance) for a given mass deposition rate on the filter, the fact that starting voltage did decrease substantially between the two studies might signal potential issues that should be addressed upon further instrument development (e.g., light leakage around the optical sensor, sensor or laser degradation, changes in the filter tape quality).

\section{Field Testing}

Fig. 7 shows time-series data from the OC-EC field analyzer, AE33 and Airwatch over all five weeks of monitoring. For the OC-EC analyzer, there is one data point per hour, for which the EC concentration value was derived from the single 30-minute sample collected during that hour. For the AE33 and Airwatch, data are shown as a 5-minute rolling average of EC concentration. The AE33 BC concentration was converted to EC using the 1.86 factor shown in Fig. 4. The Airwatch EC concentration values were determined using the calibration curve shown in Fig. 6. Where gaps exist in the time-series data, one or more of the monitors were not gathering data (i.e., due to the AE33 or Airwatch running out of filter tape, the OC-EC analyzer running out of analysis gases, or loss of mine power to the instruments). While the EC concentrations in the monitoring location of the study mine were relatively low during the field testing (i.e., generally between 10 and $120 \mu \mathrm{g} \mathrm{m}^{-3}$ per the OC-EC field analyzer data), they are certainly within a range of interest for many mine monitoring applications, where the objective is to catch an impending exceedance of a particular threshold value.

From Fig. 7, it is evident that the data from the OC-EC analyzer and the AE33 monitor track quite well together and exhibit a pattern consistent with expectations based on activities in the mine. Characteristically, the EC concentration tends to taper off over weekends and picks back up with regular production activities each Monday. However, the ratio between the OC-EC analyzer and AE33-derived EC values is not constant. Rather, there tend to be relatively long periods of time (i.e., generally a day or more) where the two EC concentrations either match as expected or the AE33 is significantly below the OC-EC value. No conclusive explanation could be determined for this behavior. It does not appear to be an issue of relative EC concentration in the sampling environment, as periods of both agreement and disagreement coincide with periods of lower and higher EC in Fig. 7. It is possible that some particular condition(s) in the mine environment (e.g., humidity or pressure) affected one of the instruments, or that one or both periodically suffered from internal problems. For instance, premature EC evolution could have occurred in the OC-EC analyzer (e.g., due to leaks in gas lines or even small amounts of mineral dust in the sample stream). It is also possible that the AE33 to OC-EC calibration factor derived from the laboratory testing (i.e., with fresh DPM and no other particulates) did not hold for some mine conditions (e.g., periods where DPM in the sampling area was relatively aged or sourced from specific equipment).

The severe oscillations in the Airwatch data in Fig. 7 are primarily attributed to the non-ideal absorbance behavior with sample loading on each filter spot. Because absorbance values were translated to EC using the linear trend shown in Fig. 6, the steep decline in absorbance followed by stabilization on each filter spot yields a similar trend for EC (compare Fig. 1 with Fig. 7 inset). Even so, during many periods, the average Airwatch-derived EC tracks well with the other two monitors. During periods where this was not the case (e.g., late in weeks 1 and 4), the exact reasons were not determined. However, the Airwatch was observed to display an error message on two occasions related to malfunction of its filter tape clamping mechanism (i.e., which allows the tape to self-advance). The first occasion was on the Monday of week 4, at which time the instrument was reset. It then appears to have functioned normally through about Tuesday of that week based on Fig. 7. When the instrument was checked the following Monday (week 5), it was again displaying the error message.

To further evaluate the correlation between the three instruments, Fig. 8 shows a comparison of the AE33 and Airwatch data against the OC-EC analyzer across the entire field study. To construct this plot, TWA values for EC concentration were determined for each AE33 or Airwatch filter spot that collected data during the study. Then, corresponding data points for the OC-EC analyzer were determined as TWA values for the same time period covered by each filter spot. The relatively high $\mathrm{R}^{2}$ value $(0.934)$ for the trend line associated with the AE33 versus OC-EC analyzer data points reflects the clear correlation between these instruments, also seen in Fig. 7. However, the relatively low slope (0.74) reflects the periodic reduction in the AE33 to OC-EC calibration factor discussed above.

Fig. 8 shows more scatter in the data points around the trend line comparing the Airwatch monitor to the OC-EC analyzer $\left(\mathrm{R}^{2}=0.845\right)$, as well a tendency for the Airwatchderived EC concentration values to be greater than those of the OC-EC analyzer (slope of 1.18). As discussed earlier, the data scatter is largely attributed to the non-ideal behavior in light absorbance during sample loading on each filter spot. Since the length of time required for the absorbance (and hence EC values) to stabilize can vary somewhat between filter spots (e.g., see Fig. 7 inset), the impact on the TWA EC values shown in Fig. 8 is variable. Aside from resolving the aforementioned mechanical issues with the Airwatch (e.g., filter clamping mechanism, factors that may affect the 


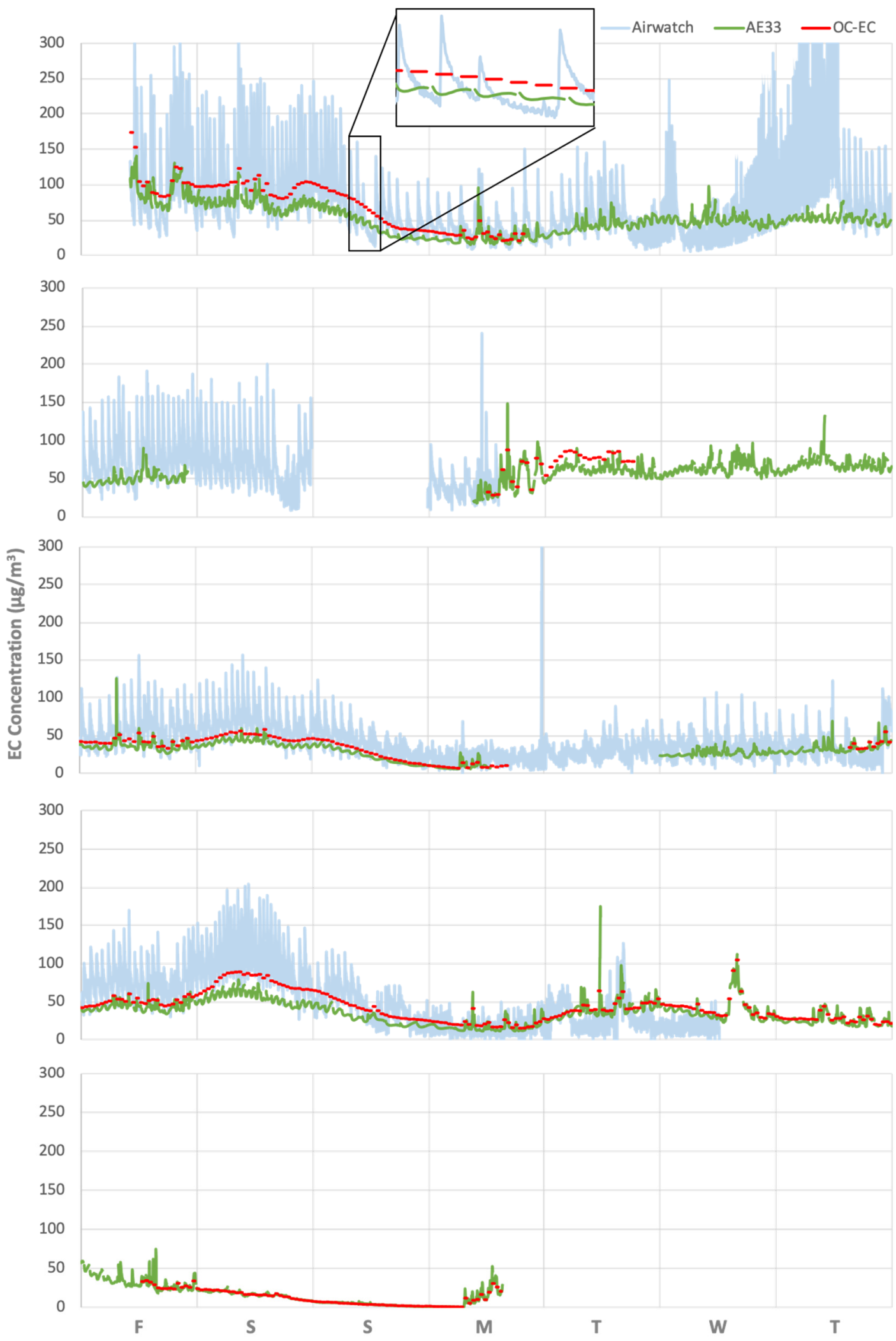

Fig. 7. Time-series EC concentration data derived from the OC-EC field analyzer, AE33, and Airwatch over the 5-week field testing (weeks 1-5 shown from top to bottom, respectively). Each 30-minute OC-EC analyzer sample value was applied across the entire hour surrounding sample collection. AE33 EC concentration was determined by multiplying BC concentration by the 1.86 factor found in the laboratory tests. Airwatch EC concentration was determined using the calibration curve developed from the laboratory test results. AE33 and Airwatch data are shown on a 5-minute rolling average basis. 


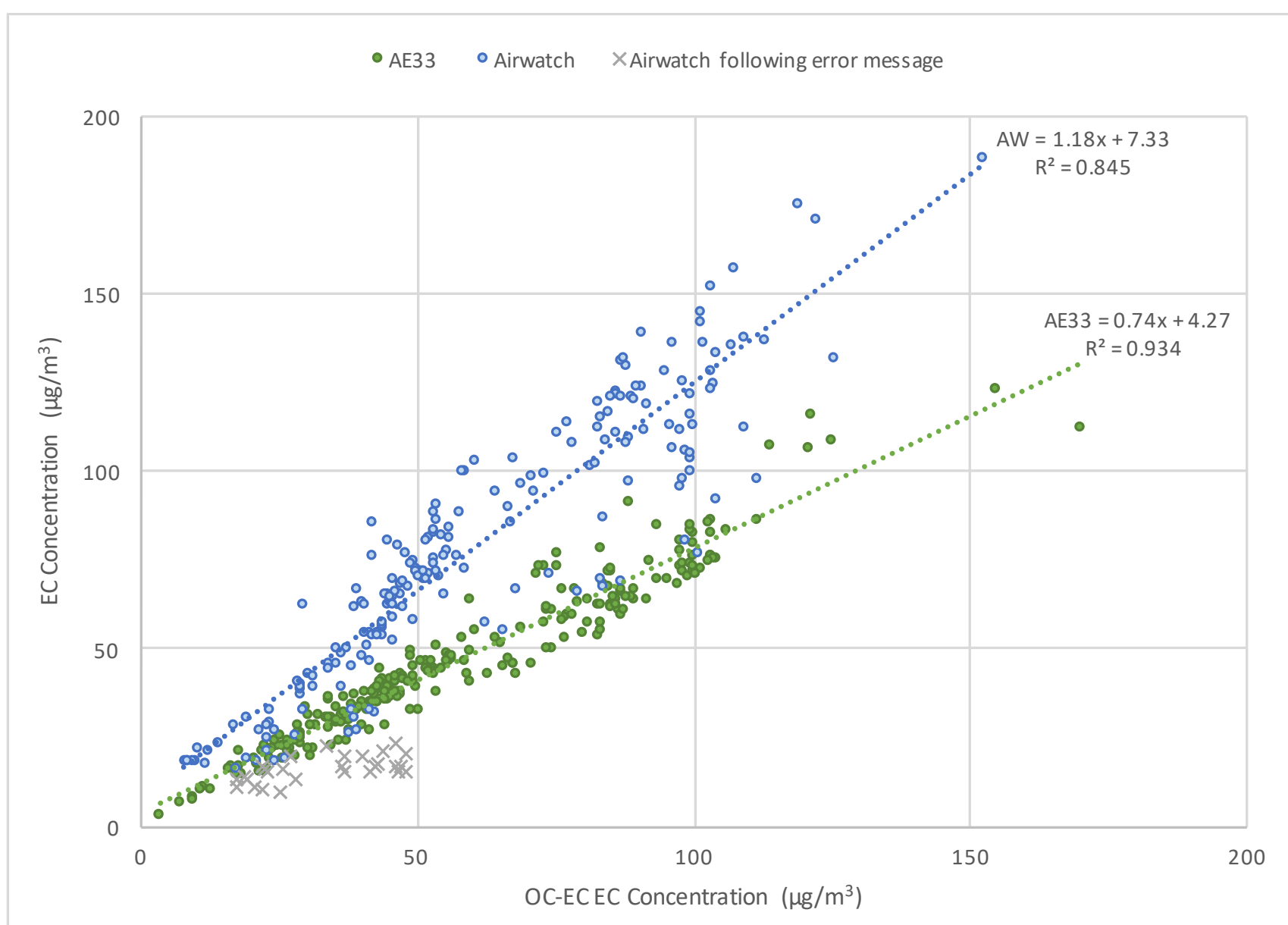

Fig. 8. AE33 and Airwatch derived EC concentration versus OC-EC analyzer EC concentration across the 5-week field study. Each AE33 or Airwatch data point represents the TWA value for a given filter spot, and the corresponding OC-EC analyzer value was determined as a TWA for the same time period. Airwatch data collected after the instrument was found to be displaying an error message late in week 4 are shown as a separate data series and were not used to construct the Airwatch trendline.

optical sensor voltage on a clean filter spot), if this instrument is to be further developed, it is clear that selection of a favorable filter tape material and development of appropriate data corrections will be critical. It is possible the filter tape tested here and in the previous Barrett et al. (2017) study could be successfully used, but more calibration data is needed to establish a robust correction that accounts for the non-ideal absorbance behavior. Alternative filter materials, and their potential challenges and benefits, should also be considered.

\section{CONCLUSIONS}

Capabilities for continuous or semi-continuous DPM monitoring in underground mines would be valuable in terms of both worker health protection and engineering purposes. Overall, the laboratory and field testing data collected in this study indicate that the commercially available OC-EC field analyzer and AE33 aethalometer could provide for longterm and autonomous monitoring under certain conditions (e.g., access to consistent power; routine maintenance to clean sampling trains and replace consumables). The prototyped Airwatch monitor also showed potential, although a number of improvements and further calibration work are needed to ensure consistent and reliable measurements.

Monitor selection would require specific consideration as to the intended application and sampling environment, including the required data collection rate and quality, and the expected EC concentrations and possible analytical interferences (i.e., due to co-occurring aerosols).

\section{ACKNOWLEDGMENTS}

The authors wish to thank the National Institute for Occupational Safety and Health for funding this work (contract number 200-2014-59646). We would also like to thank all personnel at the study mine who graciously provided their time and support for this work. At Virginia Tech, we thank Forest Wright for help with data collection and analysis, and Chreston Miller for help with Airwatch data processing. Finally, we thank Tony Hansen at Magee Scientific for technical assistance with the AE33 Aethalometer, 
and Bob Cary, Josh Dixon, and Josh Vinson at Sunset Laboratory for their assistance with the OC-EC Field Analyzer.

\section{DISCLAIMER}

The findings and conclusions in this paper are those of the authors and do not necessarily represent the official position of the National Institute for Occupational Safety and Health, Centers for Disease Control and Prevention. Mention of any company or product does not constitute endorsement by NIOSH.

\section{SUPPLEMENTARY MATERIAL}

Supplementary data associated with this article can be found in the online version at http://www.aaqr.org.

\section{REFERENCES}

Barrett, C., Gaillard, S. and Sarver, E. (2017). Demonstration of continuous monitors for tracking DPM trends over prolonged periods in an underground mine. Proceedings of the $16^{\text {th }}$ North American Mine Ventilation Symposium, pp. 5.29-5.36.

Birch, E. (2003). NIOSH Method 5040: Diesel as Particulate Matter (as Elemental Carbon) (Issue 3). U.S. Department of Health and Human Services, Centers for Disease Control and Prevention, National Institute for Occupational Safety and Health, USA.

Borak, J., Sirianni, G., Cohen, H., Chemerynski, S. and Wheeler, R. (2003). Comparison of NIOSH 5040 method versus Aethalometer to monitor diesel particulate in school buses and at work sites. AIHA J. 64: 260-268.

Cauda, E., Sheehan, M., Gussman, R., Kenny, L. and Volkwein, J. (2014). An evaluation of sharp cut cyclones for sampling diesel particulate matter aerosol in the presence of respirable dust. Ann. Occup. Hyg. 58: 9951005.

Cauda, E.G., Ku, B.K., Miller, A.L. and Barone, T.L. (2012). Toward developing a new occupational exposure metric approach for characterization of diesel aerosols. Aerosol Sci. Technol. 46: 1370-1381.

Drinovec, L., Mocnik, G., Zotter, P., Prévôt, A.S.H., Ruckstuhl, C., Coz, E., Rupakheti, M., Sciare, J., Müller, T., Wiedensohler, A. and Hansen, A.D.A. (2015). The "dual-spot" Aethalometer: An improved measurement of aerosol black carbon with real-time loading compensation. Atmos. Meas. Tech. 8: 1965-1979.

Gaillard, S., McCullough, E. and Sarver, E. (2016). Area monitoring and spot-checking for DPM in an underground mine. Min. Eng. 68: 57-63.

Janisko, S. and Noll, J.D. (2008). Near real-time monitoring of diesel particulate matter in underground mines. Proceedings of the $12^{\text {th }}$ North American Mine Ventilation Symposium, pp. 509-513.

Jimenez, J., Claiborn, C., Larson, T., Gould, T., Kirchstetter, T.W. and Gundel, L. (2007). Loading effect correction for real-time aethalometer measurements of fresh diesel soot.
J. Air Waste Manage. Assoc. 57: 868-873.

Kittelson, D.B. (1998). Engines and nanoparticles: A review. J. Aerosol Sci. 29: 575-588.

Marple, V.A.K. and Rubow, L. (1983). An aerosol chamber for instrument evaluation and calibration. AIHA J. 44: 361-367.

Mine Safety and Health Administration (MSHA) (2001). 30 CFR 57: Diesel particulate matter exposure of underground metal and nonmetal miners; final rule. 66 Fed. Reg. 5706.

Mine Safety and Health Administration (MSHA) (2006). $30 \mathrm{CFR}$ 57: Diesel particulate matter exposure of underground metal and nonmetal miners; final rule. 71 Fed. Reg. 28923.

Noll, J.D. and Janisko, S. (2013). Evaluation of a wearable monitor for measuring real-time diesel particulate matter concentrations in several underground mines. J. Оссир. Environ. Hyg. 10: 716-722.

Noll, J.D., Janisko, S. and Mischler, S.E. (2013). Real-time diesel particulate monitor for underground mines. Anal. Methods 5: 2954-2963.

Nomadics, Inc. (2013). Autonomous Networked EC Monitor, Final Report for Contract 200-2010-36901 submitted to the U.S. Department of Health and Human Services, Centers for Disease Control and Prevention, National Institute for Occupational Safety and Health, USA.

Ristovsky, Z.D., Miljevic, B., Surawski, N.C., Morawska, L., Fong, K.M., Goh, F. and Yang, I.A. (2012). Respiratory health effects of diesel particulate matter. Respirology 17 : 201-212.

Sunset Laboratory, Inc. (2014). Organic Carbon and Elemental Carbon Field Instrument (Model 4) User's Manual. Hillsborough, NC.

Volkwein, J., Barrett, C., Sarver, E. and Hansen, A.D.A. (2017a). Application of an Environmental 'Black Carbon' Particulate Sensor for Continuous Measurement of DPM in Three Underground Mines. Proceedings of the the Australian Mine Ventilation Conference, Paper No. 96.

Volkwein, J., Robertson, K. and Hansen, A.D.A. (2017b). Long-term continuous measurement of diesel particulate matter patterns in a large underground metal mine. Proceedings $16^{\text {th }}$ North American Mine Ventilation Symposium, pp. 5.19-5.27.

Volkwein, J., Vinson, R.P., Page, S., McWilliams, L.J., Joy, G.J., Mischler, S.E. and Tuchman, D.P. (2006). Laboratory and Field Performance of a Continuously Measuring Personal Respirable Dust Monitor. U.S. Department of Health and Human Services, Centers for Disease Control and Prevention, National Institute for Occupational Safety and Health, Publication No. 2006-145, Report of Investigations 9669, pp. 1-55.

Weingartner, E., Saathoff, H., Schnaiter, M., Streit, N., Bitnar, B. and Baltensperger, U. (2003). Absorption of light by soot particles: determination of the absorption coefficient by means of aethalometers. J. Aerosol Sci. 34: 1445-1463.

Yu, C.H., Patton, A.P., Zhang, A., Fanac, Z., Weisel, C.P. and Lioy, P.J. (2015). Evaluation of diesel exhaust 
continuous monitors in controlled environmental conditions. J. Occup. Environ. Hyg. 12: 577-587.
Received for review, June 242019 Revised, September 5, 2019 Accepted, September 21, 2019 